Revista Brasileira de Tecnologias Sociais | ARTIGos | DOI: 10.14210/RBTS.v5N1.P10-21

\title{
REVISÃO SISTEMÁTICA SOBRE A EFICÁCIA DE ESTRATÉGIAS NÃO FARMACOLÓGICAS NO TRATAMENTO DA ANSIEDADE: USO DA MUSICOTERAPIA E DA ACUPUNTURA
}

\author{
SYSTEMATIC REVIEW OF THE EFFECTIVENESS \\ OF NON-PHARMACOLOGICAL STRATEGIES \\ IN THE TREATMENT OF ANXIETY: USE OF \\ MUSICOTHERAPY AND ACUPUNCTURE
}

\author{
Luisa Lubian $^{1}$ \\ Carina Nunes Bossardi ${ }^{2}$
}

RESUMO: A ansiedade é um fator que acomete diversas pessoas na atualidade. O objetivo deste estudo é analisar os efeitos de duas técnicas - musicoterapia e acupuntura - como estratégias no tratamento da ansiedade, por meio de estudos empíricos acerca do tema. Para avaliar o contexto 'ansiedade', foram utilizados os descritores "ansiedade e acupuntura" e "ansiedade e musicoterapia" nas bases LILACS e SciELO, com publicações de 2007 a 2017. Foi feita uma tabela, avaliando os tipos de pesquisa, os participantes, o objetivo e o enfoque da pesquisa, os métodos utilizados e os principais resultados. Constatou-se eficácia estatisticamente significativa na maioria dos artigos analisados, de forma parcial ou definitiva. Entretanto, houve carência de dados por parte da musicoterapia, o que indica a necessidade de maiores estudos científicos na área, tendo em vista que podem ser importantes estratégias para a redução da ansiedade, como comprovado nos artigos analisados, principalmente na ala oncológica e da fibromialgia. Espera-se que os resultados dessa pesquisa possam gerar reflexões ao campo do tratamento da ansiedade e contribuições às práticas e às estratégias de saúde, visando proporcionar um tratamento complementar eficaz e que melhore a qualidade de vida da população.

PALAVRAS-CHAVE: Ansiedade; Acupuntura; Ações não farmacológicas; Musicoterapia.

ABSTRACT: Anxiety is a factor that affects many people today. The objective of this study is to analyze the effects of two techniques - music therapy and acupuncture - as strategies in the treatment of anxiety, through empirical studies on the theme. To evaluate the 'anxiety' context, the descriptors 'anxiety and acupuncture' and 'anxiety and music therapy' were used as search terms, in the LILACS and SciELO databases, searching only on publications from 2007 to 2017. A table was created to evaluate the types of research, the participants, the objective and focus of the research, the methods

Licença CC BY:

Artigo distribuído sob os termos Creative

Commons, permite uso e distribuição irrestrita em qualquer meio desde que $o$ autor credite a fonte original. used, and the main results. Statistically significant efficacy was found in most of the articles analyzed, either partially or definitively. However, there was a lack of data for music therapy, indicating a need for further scientific studies in the area, considering

1 Acadêmica do Curso de Psicologia da Universidade do Vale do Itajaí - UNIVALI. E-mail: luisalub92@gmail.com.

2 Professora do Curso de Psicologia e do Mestrado em Saúde e Gestão do Trabalho da Universidade do Vale do Itajaí - UNIVALI. E-mail: carinabossardi@univali.br. 
that strategies for anxiety reduction may be important, as evidenced in the articles analyzed, especially in the oncology and fibromyalgia ward. It is hoped that the results of this research will stimulate reflections in the field of anxiety treatment and contributions to health practices and strategies, aiming to provide an effective complementary treatment and that improves the quality of life of the population.

KEYWORDS: Anxiety; Non-pharmacological actions; Acupuncture; Music therapy.

\section{INTRODUÇÃO}

A ansiedade é um fator que acomete diversas pessoas na atualidade, sujeita a causar patologias graves. Seus níveis no organismo ocorrem em situações inesperadas, em que o corpo reage diante da surpresa e de um evento desencadeador. Desta forma, os sintomas podem estar presentes, independente de tais patologias. Entretanto, quando na forma patogênica, a ansiedade interfere nas atividades cotidianas do ser humano, ocasionando em problemas para lidar com tais situações, diante dos pensamentos de medo, de angústia e de culpa, característicos do transtorno (CASTILLO; RECONDO; ASBAHR; MANFRO, 2000).

Segundo o Manual Estatístico de Transtornos Mentais - DSM-5 (APA, 2014), a definição para ansiedade seria a antecipação do futuro e o medo em resposta emocional a ameaças reais ou não. Os dois seriam diferentes, pois o medo se daria sobre comportamentos de fuga e a ansiedade seria a vigília de algo que irá acontecer, resultando em comportamentos de esquiva. Seus transtornos se diferenciam diante das condições que causam o medo e o comportamento esquivo. Esses transtornos podem se desenvolver na infância e persistirem na idade adulta, caso não haja tratamento e reconhecimento da doença, sendo que a estimativa é de maioria no sexo feminino.

Para determinar se a ansiedade é de cunho patológico, é necessária uma avaliação clínica, bem como observar a frequência dos pensamentos e se eles prejudicam nas tarefas cotidianas, levando em conta o contexto cultural e os grupos sociais. O diagnóstico ocorre quando se constata não haver efeitos de usos medicamentosos ou que não são explicados por outro transtorno mental (APA, 2014).

Sabendo, portanto, dos diferentes graus de ansiedade, a medicina utiliza de estratégias farmacológicas para poder controlar os efeitos negativos que ela causa por meio de medicamentos específicos e receitados por psiquiatras, de acordo com o diagnóstico recebido. A respeito das terapias farmacológicas, Braz et al. (2011) afirmam que, além dos benzodiazepínicos e antidepressivos, existem os moduladores dos canais de cálcio, relaxantes musculares e analgésicos, sendo que, para os autores, estes muitas vezes podem não trazer resultados eficazes ou ocasionam efeitos colaterais. Desta forma, os usuários buscam a solução diante das terapias complementares.

As terapias complementares são estratégias não farmacológicas, de cunho científico ou não, que têm sido praticadas por algumas pessoas e tidas como soluções amenas para diversas enfermidades, dentre elas, a ansiedade. Dentre essas estratégias, pode-se citar a dança, a pintura, a meditação, as técnicas de relaxamento, a massagem, dentre outros (BRAZ et al., 2011). Para tanto, este trabalho terá como foco o uso de estratégias não farmacológicas no tratamento da ansiedade, especialmente no que se refere aos efeitos da musicoterapia e da acupuntura.

Assim sendo, existem medidas integrativas que auxiliam na redução da ansiedade e que são utilizadas no Sistema Único de Saúde (SUS) e em diversos contextos. A acupuntura é uma destas técnicas, trazida pela Organização Mundial em Saúde (OMS) como uma das práticas que 
podem ser utilizadas; enquanto que a musicoterapia é vista nas pesquisas como uma estratégia para auxiliar no tratamento de diversas enfermidades (CARNEVALE et al., 2017)

A respeito da acupuntura, Rocha, Benedetto, Fernandez e Gallian (2015) trazem que a acupuntura é um instrumento de intervenção no ramo da saúde abordando o processo saúdedoença, sendo usada tanto de forma isolada quanto junto a outros procedimentos, de forma rápida e barata, conforme também apontam Kurebayashi et al. (2017). A acupuntura é reconhecida desde 1995 como uma das práticas integrativas no SUS (ROCHA et al., 2015).

A outra técnica avaliada nesse estudo será a musicoterapia. Esta é, segundo Zanini et al. (2009), definida como o uso da música e seus elementos, como sons, ritmo, harmonia, dentre outros, por um profissional musicoterapeuta qualificado para tal, dentro de um grupo ou individualmente, buscando construir um processo terapêutico por meio das técnicas da música. A musicoterapia teve início de sua prática no século $\mathrm{XX}$, ascendendo de forma relevante diante dos estudos e pesquisas atuais, que buscam compreender seu uso, sendo reconhecida como expressão subjetiva tanto no musicoterapeuta como daqueles que estão dentro do grupo (CUNHA; VOLPI, 2008).

Ainda segundo Cunha e Volpi (2008), quanto às práticas da musicoterapia, essas podem ser vistas dentro de escolas, clínicas, hospitais, empresas e vários órgãos públicos e privados, com diversas populações, sendo que seus estudos trazem relevância tanto em pacientes oncológicos como em idosos. Cunha e Volpi (2008) trazem sua relevância no âmbito escolar, como estímulo às habilidades cognitivas e de aprendizagem. No âmbito hospitalar, atua na recuperação tanto emocional como física dos pacientes. E no âmbito social, é vista como uma estratégia para auxiliar na promoção da saúde e na busca da história de vida por meio da música nos grupos e na Alta Complexidade e em locais de apoio como asilos e presídios.

O sujeito que sofre da ansiedade tem dificuldades em sua rotina diante do desconforto, que causa situações inesperadas e seus medos, dificultando seu convívio com o contexto em que está inserido. Por meio das estratégias anteriormente mencionadas, ele pode se sentir mais confiante e mais capaz de enfrentar essas situações, uma vez que os efeitos da musicoterapia e da acupuntura proporcionam o bem-estar social (SILVA; TESSER, 2013). A relevância das estratégias não farmacológicas está presente no SUS, e Silva e Tesser (2013) trazem que o contato com a acupuntura incentiva uma postura ativa e uma visão dos pacientes sobre o cuidado com o corpo, tornando-se 'mais em conta' que muitos medicamentos tradicionais.

A acupuntura pode servir tanto como tratamento principal como complementar de diversas doenças, tais como: enxaquecas, alergias, ansiedade, estresse e depressão. Além do que os achados demonstram a influência profunda da acupuntura diante de problemas físicos e emocionais, bem como ser uma prática integrativa e complementar (SANTOS; GOUVEIA; MARTELLI; VASCONCELOS, 2009, p. 331). Lin, Hsing e Pai (2006) trazem que a acupuntura está sendo cada vez mais divulgada no âmbito médico e tendo sua eficácia comprovada, mudando seus profissionais e sua forma de prática, como em locais de ensino, tanto no Brasil como em outros países.

Para Firmeza et al. (2017), o uso da música é descrito em diferentes publicações, tendo efeitos em diversas áreas do corpo, tanto nos batimentos cardíacos, como na redução da dor. Alguns autores afirmam que a música gera um efeito terapêutico e de prazer, porque se relaciona ao afeto e às emoções. Em alguns escudos, como o de Oliveira et al. (2012), o efeito da musicoterapia é relevante em idosos com Alzheimer e com Parkinson; outro estudo de Tudissaki (2014) afirma que há uma escala da musicografia em braile. 
Diante do exposto, este estudo tem por objetivo analisar os efeitos dessas duas técnicas - musicoterapia e acupuntura - como estratégias no tratamento da ansiedade, por meio de estudos empíricos acerca do tema. Pretende-se, em especial, conhecer a metodologia utilizada pelas pesquisas, bem como os principais resultados encontrados. Espera-se que os resultados dessa pesquisa possam gerar reflexões ao campo do tratamento da ansiedade e contribuições às práticas e às estratégias de saúde, visando proporcionar um tratamento complementar eficaz e que melhore a qualidade de vida da população.

\section{METODOLOGIA}

O presente artigo trata-se de uma revisão sistemática da literatura, utilizada como estratégia para a busca de pesquisas empíricas que investigaram a eficácia dos métodos não farmacológicos no tratamento da ansiedade, sendo tais métodos representados nesse estudo pelas práticas integrativas da musicoterapia e da acupuntura. Para a coleta de dados, utilizou-se as bases de dados on-line que foram acessadas por meio da Biblioteca Virtual em Saúde - Psicologia (BVSPsi- http://www.bvs-psi.org.br).

As bases de dados escolhidas foram SciELO (Scientific Electronic Library On-line) e LILACS (Literatura Latino-Americana e do Caribe em Ciências da Saúde). As palavras-chave foram "acupuntura" "musicoterapia” e "ansiedade”, todos os descritores localizados no índice de terminologias da BVS-Psi. Nesse sentido, a palavra "ansiedade" foi combinada por meio do critério "AND” às palavras "acupuntura" e "musicoterapia”. Foram incluídos artigos científicos empíricos, com textos completos disponíveis, com ano de publicação entre 2007 a 2017 e no idioma português, Brasil.

\section{RESULTADOS E DISCUSSÃO}

No sítio da LILACS, as palavras-chave para “ansiedade e acupuntura” obtiveram 256 resultados e, com refinação e critérios de exclusão, restaram 10 resultados. Para as palavras “ansiedade e musicoterapia”, foram encontrados 289 resultados e, com a refinação, sobraram 03 resultados. No sítio da SciELO, as palavras-chave "ansiedade e acupuntura” geraram 13 resultados e, com a refinação e critérios de exclusão, ficaram 03 resultados. Para as palavras "ansiedade e musicoterapia”, houve 06 resultados e, com a refinação, obteve-se um (0 1) resultado. Os critérios de exclusão utilizados foram as revisões de literatura, as publicações com mais de dez anos, as que não tivessem versão em português e que se encaixassem em publicações como teses, livros ou outros textos acadêmicos. Nessa análise, foram excluídos também os artigos repetidos.

No total, portanto, juntando os resultados obtidos nas bases SciELO e LILACS, os descritores "ansiedade e acupuntura" obtiveram 13 resultados e "ansiedade e musicoterapia" O4 resultados, considerando os critérios de exclusão e refinação. Após a leitura dos resumos dessas pesquisas, foi possível constatar que 08 artigos se tratavam de revisões da literatura. Para finalidade dessa pesquisa, esses estudos foram excluídos da amostra de análise. Sendo assim, foram analisados, na íntegra, 06 artigos sobre ansiedade e acupuntura e 03 artigos a respeito de ansiedade e musicoterapia. Nessa leitura, as pesquisas foram avaliadas segundo autores e ano; título; objetivo; participantes; método e instrumentos; tipos de pesquisa e principais resultados. A seguir, serão descritos os resultados encontrados para as terapias não farmacológicas acupuntura e musicoterapia em relação ao tratamento da ansiedade. 


\subsection{EFICÁCIA DA ACUPUNTURA NO TRATAMENTO DA ANSIEDADE}

Serão apresentados os resultados das 06 pesquisas relacionadas à acupuntura.

Constatou-se que os artigos pesquisados tiveram diversificados anos de publicação datados de 2007, 2008, 2009, 2010, 2014 e 2015. Outro fato visto é a presença do tema "fibromialgia", presente em dois dos seis artigos, pelos autores Stall et al. (2015) e Takiguchi et al. (2008). O primeiro por meio da diferença do método de Rolfing e acupuntura e o segundo, sobre os efeitos na melhora da qualidade de vida.

Stall et al. (2015), em sua pesquisa, tiveram como objetivo avaliar o efeito do Rolfing3 e acupuntura, individual ou de forma conjunta. Dessa maneira, não foi necessário, segundo o autor, a inclusão de grupos controle com método placebo. O método utilizado foi um estudo comparativo, prospectivo e complementar ao tratamento farmacológico. Para poder fazer o procedimento, foram feitas 10 sessões com o método Rolfing ${ }^{3}, 10$ sessões de acupuntura e 10 sessões com Rolfing e acupuntura, bem como o uso de escalas como o Inventário de Ansiedade de Beck (BAI) e o Inventário de Depressão de Beck (BDI). O público-alvo foi de 60 sujeitos - tendo em cada grupo 20 voluntários que aderiram ao tratamento - todos com mais de 18 anos e portadores de síndrome fibromiálgica (SFM) diagnosticados por um médico neurologista. Os resultados encontrados na pesquisa foram a melhora nos três grupos, em destaque para os resultados da acupuntura, que apresentou valores mais elevados em comparação a outros grupos, tendo diferenças estatisticamente significativas.

Já na pesquisa de Silva (2010), num estudo individual em apenas uma paciente, foi realizado o tratamento da ansiedade pela acupuntura por meio de um estudo de caso. O objetivo deste trabalho pautou-se no tratamento realizado com uma paciente com transtorno de ansiedade, diante de 10 sessões. A paciente do estudo possuía 39 anos, era casada e residente em uma cidade baseada na agricultura. O motivo trazido foi, segundo ela, por apresentar um constante estado de ansiedade aliada à depressão leve, sendo que há nove anos tomava antidepressivos e fora diagnosticada com quadro de depressão, sendo que, mais adiante, fora confirmado o diagnóstico de Transtorno de Ansiedade Generalizada. O método usado foi a punção nos pontos de acupuntura, sendo eles o R3, R6, C7, CS6, CS7, E25, E36, P5, P7, P9, IG4, IG1 1, F3, F14, VC4, VC6. VC12, VC15, VC17, BP3, BP6, BP9, TA4, TA5 e Yintang. Para a seleção e a utilização desses pontos, foi feita uma investigação com o paciente e exame dos pulsos e da língua para verificar o andamento do tratamento. Os resultados encontrados foram a redução da intensidade dos sintomas, mas não considerado uma cura definitiva. Mesmo quando não apresentou mais os sintomas, a paciente continuou fazendo o acompanhamento da acupuntura mensalmente para a manutenção.

Por sua vez, a pesquisa de Takiguchi (2008) retrata a melhora da qualidade de vida em pacientes com fibromialgia fazendo uso da acupuntura. O objetivo do estudo foi pautado na eficácia desse método na vida dos pacientes e sua melhora na dor, sono e qualidade de vida de mulheres fibromiálgicas. O método utilizado para avaliação foi um ensaio clínico randômico, com inserção das agulhas tenderpoints na base occipital. Para avaliar a dor, foi utilizada a escala visual e analógica de dolorimetria, o sono pelo Inventário do sono e qualidade de vida pelo Questionário de Impacto na Fibromialgia (QIF). Foram utilizados dois grupos, cujo público- alvo eram 12 mulheres com idade de 44 e 45 anos, diagnosticadas com fibromialgia, cujas sessões ocorreram em um período de

3 Método criado pela bioquímica norte-americana Ida Pauline Rolf. Busca melhorar a qualidade do equilíbrio e do alinhamento do corpo humano por meio do aperfeiçoamento dos movimentos. 
oito semanas. Os resultados apontam melhora principalmente quando utilizados os tenderpoints no grupo, entretanto a diferença estatística significante se encontrou apenas no grupo B diante das três intervenções e, no grupo A, observaram-se mudanças, porém menores que as do grupo B e não estatisticamente significativas, sendo que o grupo B demonstrou diminuição no limiar da dor.

Já Caires e colaboradores (2014) trazem uma pesquisa sobre a utilização de terapias complementares nos cuidados paliativos, tendo dentre elas a acupuntura. O objetivo dessa pesquisa pautou-se na utilização das terapias complementares nos cuidados paliativos por meio de instituições brasileiras credenciadas nas Associações Nacionais e Latino-Americana de Cuidados Paliativos. O método utilizado para essa pesquisa foi exploratório e descritivo, com abordagem qualitativa e quantitativa. Os participantes voluntários eram provenientes de instituições hospitalares, domiciliares e serviços que utilizam das terapias alternativas e que aceitaram a participação na pesquisa. Para coletar os dados e recrutar voluntários, foi-se utilizado um questionário semiestruturado com questões objetivas e subjetivas, enviado por correio eletrônico, sendo que aqueles que responderam o formulário foram: dois médicos, dois enfermeiros, um fisioterapeuta e um administrador - obtendo-se, assim, seis participantes. Os resultados encontrados foram que todas as instituições referenciaram a musicoterapia e a acupuntura citada por 4 dos 6 participantes dentre as terapias, sendo que as terapias complementares mais utilizadas foram a massagem, a acupuntura e a musicoterapia. Os relatos trazidos pelos voluntários são os efeitos das terapias complementares aliados ao tratamento convencional farmacológico e que estes proporcionam melhor controle de sintomas psicológicos, físicos e emocionais na ansiedade, na depressão e na dor.

Haddad, Oliveira, Simões e Marcon (2009) trazem uma pesquisa sobre mães lactantes de recém-nascidos com baixo peso. Nessa pesquisa, o objetivo foi relatar a experiência da utilização da acupuntura no estímulo à lactação de mães de bebês de muito baixo peso. O método utilizado foi a quiroacupuntura, uma forma da utilização da acupuntura nos pontos das mãos. O público-alvo da pesquisa foram mães lactantes na Unidade de Terapia Intensiva Neonatal - no município de Londrina, sendo realizada nos meses de julho e agosto de 2007. Quanto aos resultados, observouse que as mães, quando questionadas sobre como se sentiram em comparação à semana anterior da sessão, trouxeram como relatos indicadores de bem-estar e de melhora. A liberação de ocitocina por efeito da técnica de quiroacupuntura traz a diminuição da tensão e ansiedade.

E, por fim, Kurebayashi, Freitas e Oguisso (2009) trabalham com enfermidades tratáveis com a acupuntura segundo a percepção de enfermeiras. O objetivo deste trabalho se pauta na discussão e na análise das enfermeiras diante da acupuntura e da sua aplicabilidade, por meio da listagem de doenças tratadas pela acupuntura, publicadas em 2003. Os participantes dessa pesquisa foram 33 enfermeiras de 11 Unidades de Saúde Pública do município de São Paulo. O método utilizado foi um estudo exploratório e foi realizada uma entrevista com 3 questões: 1) Você já se tratou com acupuntura? 2) Se você não se tratou, se trataria e com que finalidade? 3) Em que casos você iria sugerir a acupuntura? As entrevistas tiveram a duração de junho a julho de 2007. Os resultados trazidos segundo os dados estatísticos foram sugestões da acupuntura para dores musculares, articulares, fibromialgia e tendinite, com 41,5\% das indicações. Em segundo lugar, ficaram as doenças crônicas, diabetes, hipertensão e obesidade, com 10\% para o tratamento por meio da acupuntura, diante da vivência das enfermeiras entrevistadas. A ansiedade teve 6,1\% da aprovação, enquanto outras doenças como insônia, lúpus, tabagismo e outras tiveram resultados inferiores a $5 \%$. Dessa forma, para os autores, os resultados, que a percepção da acupuntura pelas enfermeiras não difere na listagem oferecida pela Organização Mundial da Saúde e a acupuntura, na 
ansiedade, são considerados como um tratamento à parte e com índice de aceitação dos enfermeiros inferior a doenças musculares, que tiveram maior aceitação diante das entrevistas.

Constatou-se, dentro da acupuntura, que os estudos demonstrados nesse trabalho tiveram maior eficácia no sentido analgésico em doenças musculares como fibromialgia e dores físicas. Em duas das pesquisas houve o método do Questionário de Impacto na Fibromialgia, que foi utilizado para avaliar a qualidade de vida nos pacientes e o curso do efeito da acupuntura nesses pacientes, em que todos possuíram feedback positivo, com curas parciais. Por exemplo, o estudo de Stall et al. (2015) teve maiores efeitos estatísticos do que no estudo de Silva (2010), que contou com o relato do paciente, mas que não abordou pesquisas quantitativas. Foram utilizados recursos como avaliação psicológica, feitos por Stall et al. (2015), que abordaram os testes de ansiedade e depressão de Beck. Eles tiveram resultados estatísticos significantes nos três grupos de forma quantitativa. Outra possível observação é que houve tanto pesquisa transversal quanto do tipo longitudinal, abrangendo desde estudos que implicam a investigação de algo que já foi feito, até algo feito de forma recente, demonstrando que a acupuntura possui estudos atuais e empíricos.

Além do exposto, também se pode constatar a utilização da acupuntura de forma isolada, pois geralmente ela é utilizada de forma complementar a outros tratamentos, geralmente farmacológicos para a redução dos sintomas ansiosos. Houve dois casos em que se utilizou da acupuntura de forma isolada, ou seja, sem a presença de um instrumento psicométrico ou medicamento de forma conjunta. Estas pesquisas foram o tratamento feito por Silva (2010) nos pontos da ansiedade e o trabalho com acupuntura nas mãos (quiroacupuntura) feito por Haddad, Oliveira, Simões e Marcon (2009). Também foi possível constatar que os efeitos científicos estudados pela acupuntura foram relevantes, diante de maior número de estudos e pesquisas empíricas sobre o tema.

\subsection{EFICÁCIA DA MUSICOTERAPIA NO TRATAMENTO DA ANSIEDADE}

A seguir será apresentada a análise das 03 pesquisas relacionadas à musicoterapia.

Na segunda tabela, observa-se uma discrepância em relação aos primeiros dados, em que se nota uma necessidade do levantamento de novas pesquisas sobre o tema, bem como de aprimorar os dados no assunto "ansiedade e musicoterapia", sendo este um assunto pouco retratado nas pesquisas especialmente no que se refere aos seus efeitos. Constata-se indicação para pesquisas futuras, atrelando os seus efeitos em diversos aspectos da ansiedade, como na fobia social e no transtorno de deficit de atenção e hiperatividade, sendo um dos exemplos que podem ser citados relevantes da musicoterapia no conceito ansiedade, visto que idosos com Alzheimer, como dito anteriormente, são beneficiados e estimulados mentalmente quando ouvem a música.

Observou-se que houve poucos artigos possíveis de serem analisados, visto que, dentre os critérios de exclusão utilizados, os estudos, em sua maioria, constituíam artigos de revisão de literatura, trazendo poucos dados a respeito da eficácia da musicoterapia no tratamento da ansiedade. Em relação aos anos de publicação, foram encontrados artigos em 2017, 2011 e 2009.

Na pesquisa de Franco e Rodrigues (2009), o assunto pauta-se no alívio diante da música em pacientes oncológicos com dor crônica. O seu objetivo foi de avaliar a percepção destes com dor crônica sobre os efeitos da música no alívio da dor; identificar se ocorrem alterações nos sinais vitais antes e após a sessão de musicoterapia e identificar se a intensidade da dor era reduzida após a sessão de música. Esse estudo foi realizado na unidade de oncologia de um hospital particular, de 
grande porte, localizado no município de São Paulo. A população do estudo foi composta por dez pacientes, com idade acima de 20 anos, portadores de câncer que apresentavam dor crônica. Foram realizadas entrevistas antes e após a sessão de música, e a pergunta norteadora foi "Como o senhor (a) está se sentindo neste momento?”. Para a comparação entre as variáveis: dor, frequência cardíaca (FC), respiratória (FR) antes e após as sessões de música foi utilizado o teste t de Student. Os resultados obtidos foram de que os pacientes, após as sessões de música, relataram ter obtido alívio da dor, além de relaxamento, contato com lembranças pessoais e esquecimento dos problemas. Foram identificadas alterações nos parâmetros vitais e intensidade da dor antes e após as sessões de música, com diferença estatisticamente significativa de acordo com o teste t de Student. Além da redução da dor, ocorreu redução nos valores dos parâmetros vitais, principalmente FR e FC. Dessa forma, o autor traz que essas estratégias foram possíveis de causar efeitos positivos e bons resultados estatisticamente significativos no que tange à musicoterapia na dor crônica e nos derivados, como a ansiedade.

Em Firmeza et al. (2017), o objetivo pautou-se em avaliar a ansiedade no momento do tratamento ambulatorial. Os autores trazem um estudo envolvendo pacientes com câncer de cabeça e pescoço. O método utilizado foi um ensaio clínico controlado, randomizado. O estudo foi desenvolvido em um ambulatório para tratamento de pacientes com câncer de cabeça e pescoço de um hospital universitário do estado do Ceará, Brasil. Os participantes dessa pesquisa foram pacientes em acompanhamento ambulatorial, os quais atenderam aos seguintes critérios de inclusão: com câncer de cabeça e pescoço em período pós-cirúrgico mediato; em tratamento quimioterápico ou radioterápico; com idade superior a 18 anos; e alfabetizados. A coleta de dados ocorreu no período de março a junho de 2015. Para a coleta dos dados, foram utilizados dois instrumentos: o primeiro contendo dados sociodemográficos e clínicos; o segundo foi a escala autoaplicável STAI/IDATE (State-Trait Anxiety Inventory - Inventário de Ansiedade Traço-Estado). Os resultados foram que na escala de ansiedade-estado, quando comparados com os períodos pré e pós-intervenção, todos os participantes $(100,0 \%)$ do grupo experimental apresentaram redução nos níveis de ansiedade mensurados. Isso representou uma redução média de 10,5 pontos entre os valores observados na primeira e na segunda aplicação.

Dentre os que foram submetidos à intervenção musical, $85 \%$ foram classificados com nível moderado de ansiedade antes da aplicação da música. Após intervenção, a maioria (55\%) foi classificada com nível de ansiedade baixa. Houve redução estatisticamente significante em quase todos os sinais avaliados, comparando-se com o GC. No GC, houve diminuição com significância, estatística e clínica. Os resultados não podem ser generalizados para todas as instituições, pois o estudo foi conduzido em apenas uma instituição brasileira. Apesar dessa limitação, os resultados mostram-se relevantes para o conhecimento dos enfermeiros e para a prática sobre o uso da música no controle da ansiedade em ambiente de cuidado ambulatorial. Os enfermeiros devem observar problemas decorrentes da ansiedade, identificando alterações em sua prática clínica, bem como implementar estratégias para seu controle nas diversas esferas do cuidar em oncologia.

Já no estudo de Junior e colaboradores (2012), o interesse foi de avaliar a influência da música na dor e na ansiedade de pacientes portadoras de câncer de mama, submetidas à cirurgia em um hospital em Natal (RN). O público-alvo foi constituído em dois grupos, sendo um deles submetido à intervenção por meio de música - o Grupo Experimental (GE), enquanto no outro, o Grupo Controle (GC), não houve intervenção. Ao todo, foram testados 29 pacientes, dos quais o GE foi constituído por 15 pacientes (com idade de 12 a 61 anos) e o GC por 14 pacientes (com 
16 a 55 anos). O método utilizado foi um ensaio clínico controlado, "utilizando-se randomização simples grupo experimental (GE)” (JUNIOR et al. 2012), realizado no período de agosto de 2008 a abril de 2010. O Protocolo de pesquisa utilizado consistiu na avaliação da ansiedade pelo IDATE. Nos resultados encontrados foram constatados que houve uma redução significante no escore de ansiedade IDATE-Estado. Os autores referem que a música trouxe resultados positivos da redução dos fatores da ansiedade pré-operatória, mas não de forma estatisticamente significativa, sendo necessários novos estudos.

Constatou-se, como dito anteriormente, serem necessários maiores estudos e pesquisas futuras sobre os efeitos da musicoterapia na ansiedade. Entretanto, foi possível observar nas três pesquisas analisadas a eficácia do método. Rodrigues (2009) trouxe que os resultados obtidos incluíam o alívio da dor crônica em pacientes oncológicos e da sua intensidade no corpo, acreditandose que a música foi um recuso utilizado de forma benéfica e terapêutica. O estudo presente é, segundo o autor, uma pesquisa Transversal em dez pacientes, sendo utilizada a entrevista antes e após a sessão de música, em três dias diferentes, por meio do método teste e pós-teste.

Outro ponto visto é que os três artigos estudados se tratavam dos efeitos contra a ansiedade em uma doença crônica, como o câncer. O primeiro falava dos efeitos em pacientes mulheres com câncer de mama; o segundo, dos efeitos na dor crônica em pacientes oncológicos; e o terceiro, os efeitos da música em um ambulatório para pacientes com câncer de cabeça e pescoço - sendo seu nome científico "carcinoma epidermoide da cabeça e pescoço”. Dessa forma, os três artigos presentes representam o quesito saúde-doença na ala hospitalar e oncológica. Os pacientes que obtiveram o ensaio clínico de Firmeza et al. (2017) se tratavam de pessoas acima de 18 anos, se utilizando do Grupo Controle (GC) e Grupo Experimental (GE), tornando a pesquisa de cunho Transversal, assim como no grupo de Rodrigues. Em relação à diferença dos dados, observa-se que o grupo de Junior et al. (2012) e Firmeza et al. (2017) possui o contexto da pesquisa Longitudinal prospectiva, esta sempre sendo elaborada conforme o grupo exposto e o grupo não exposto, diante de um determinado período de tempo, almejando a pesquisa por meio de teste e pós-testes para comparação dos resultados. Já Franco et al. (2009) buscou o sentido Transversal de prevalência pelo estudo ser feito em um mesmo momento histórico.

Tendo em vista os efeitos da ansiedade na vida de um indivíduo, faz-se necessário ampliar os estudos que considerem as diferentes estratégias para a eficácia do seu tratamento. É possível constatar em outras pesquisas não incluídas na análise, como a de Alves (2012) traz em seus estudos o uso da acupuntura em crianças com excesso de peso e sintomatologia da ansiedade. Para fomentar sua pesquisa, fez-se a Escala de Stress Infantil (ESI) e aparelho Ryodoraku, constatando que, num prazo de seis semanas, com o experimento, as crianças obtiveram amenização dos sintomas da ansiedade de forma parcial. Alves-Rezende e colaboradores (2012) indicaram outras fontes possíveis de ansiedade, como os efeitos possíveis na ansiedade presente, como na dor orofacial, cuja ansiedade também se faz presente e que merece investigação. Os autores constataram que os efeitos da acupuntura no sentido ansioso da área orofacial se deram de forma parcial, capaz de servir como espécie de analgésico e anti-inflamatório, por meio do aguilhoamento. Esse efeito, apesar de parcial, é também significativo para amenizar a dor crônica e tensão muscular que as disfunções temporomandibulares acarretam.

Para os estudos da musicoterapia como efeito não farmacológico no tratamento da ansiedade, Vianna e colaboradores (2011) trazem os efeitos em aleitamento materno em mães de recém-nascidos prematuros, sendo que no grupo de musicoterapia em relação ao grupo controle, o primeiro possui 
frequência maior do aleitamento materno após o tratamento. Ainda que para o autor a forma não seja estatisticamente significativa, este afirma ter influência positiva e parcial. Já Cavalcante e Pschiski (2015) trabalham com os efeitos da musicoterapia na ansiedade decorrente da hipóxia isquêmica encefálica experimental; este estudo foi feito em 24 ratas em 04 grupos. Os resultados trazidos foram que o grupo que recebeu tratamento teve efeito ansiolítico ao ouvir a música. Dessa forma, a música pôde reduzir os níveis de ansiedade nestes animais, observando que os efeitos da musicoterapia na ansiedade não estão presentes apenas em seres humanos, mas também em outros seres vivos.

E, por fim, Utida (2015) trabalha com a musicoterapia em pacientes com níveis de ansiedade e estresse com portadores de válvula cardíaca. Para fazer sua pesquisa, este se utilizou de uma escala de avaliação de estresse, assim como o inventário de ansiedade traço-estado e a presença de uma musicoterapeuta durante dez semanas. Os resultados trazidos foram a redução da ansiedade e estresse presente nos pacientes. $\mathrm{O}$ autor relata que, ao fim do trabalho, houve por parte de pacientes depoimentos de superação e resiliência diante das sessões. Demonstra-se, portanto, que a música influencia no contato afetivo e proporciona melhoras terapêuticas não somente físicas diante da redução parcial dos sintomas, mas também de forma psicológica, trabalhando com conceitos da melhora da autoestima. Sendo a música uma canalização do contato afetivo, ela proporciona efeitos diversos, além da redução parcial, portanto na ansiedade.

\section{CONSIDERAÇÕES FINAIS}

Diante desta pesquisa, observou-se a eficácia das técnicas não farmacológicas, ainda que em pequena parcela de dados. Por meio dessa revisão, foi possível observar que muitas pessoas são beneficiadas por meio das terapias complementares, podendo ser os métodos não farmacológicos eficazes no combate a doenças como câncer, fibromialgia e principalmente no que se refere à ansiedade, que pode estar presente conjuntamente a essas patologias.

Evidenciou-se a necessidade de maiores estudos tanto no quesito dos efeitos da acupuntura como da musicoterapia no contex to da ansiedade, pois há uma concentração de revisões integrativas e poucos estudos empíricos que possam projetar melhores resultados nesse trabalho. Pode-se constatar que se obtiveram poucos resultados estatísticos, considerando o número de artigos. Ainda no que se refere aos estudos empíricos a respeito do tema, a maioria dos voluntários deu feedback positivo em resposta aos dois métodos estudados.

Certificou-se, deste modo, que as terapias complementares musicoterapia e acupuntura têm sua importância e têm sido eficazes e aliadas a outras formas de tratamento, especialmente no que se refere ao tratamento farmacológico ou medicamentoso. As terapias complementares têm sido, segundo as pesquisas, muito eficazes no tratamento, proporcionando o bem-estar e o lazer, presentes não apenas em seres humanos, mas também em animais.

Desta maneira, por meio destes métodos, reconheceram-se efeitos como o bem-estar social e a redução parcial ou total dos sintomas de ansiedade, durante o período de tratamento contra seus efeitos, por meio destas técnicas não farmacológicas, sendo que esses efeitos podem não ser vistos nas primeiras sessões, ocorrendo gradativamente, mas sempre após o processo, há melhoras estatísticas significativas para a vida das pessoas que o recebem.

Dentre as limitações encontradas para este estudo, indicam-se a necessidade de projetos futuros e a ampliação do campo de pesquisas no que tange ao tema escolhido, assim como a busca 
de um período maior de preparação na pesquisa para colher os dados. Espera-se que esse estudo possa ter contribuído no sentido da relevância dessas técnicas, gerando novos dados para estudos futuros e que sirvam de base científica para constatar os benefícios das terapias complementares. Outrossim, esta revisão pode servir como indicativo aos profissionais do serviço de saúde e também à população em geral, para que conheça a eficácia das estratégias não farmacológicas como tratamento aliado às farmacologias no tratamento da ansiedade ou de outras doenças que ameacem a saúde e o bem-estar dos indivíduos.

\section{REFERÊNCIAS}

ALVES, C. P. Uso da acupuntura em crianças com excesso de peso e sintomatologia de ansiedade: um estudo exploratório. 2012. 172 f. Dissertação (Mestrado em Psicologia) - Universidade Federal de Uberlândia, Uberlândia, 2012.

ALVES-REZENDE, M. C. R. et al. Intervenção interdisciplinar no tratamento do paciente com dor orofacial: uso de acupuntura. Revista de Odontologia da Unesp, Araraquara, v. 41, p. 181, jul. 2012.

AMERICAN PSYCHIATRIC ASSOCIATION (APA). Manual diagnóstico e estatístico de transtornos mentais: DSM-5. 5. ed. Porto Alegre: Artmed, 2014.

BRAZ, A. de S. et al. Uso da terapia não farmacológica, medicina alternativa e complementar na fibromialgia. Revista Brasileira de Reumatologia, São Paulo, v.51, n.3, p.275-282, jun. 2011.

CAIRES, J. S. et al. A utilização das terapias complementares nos cuidados paliativos: benefícios e finalidades. Cogitare Enfermagem, Curitiba, v.19, n.3, p.514-520, jul./set. 2014.

CARNEVAlE, R. C. et al. O Ensino da Acupuntura na Escola Médica: Interesse e Desconhecimento. Revista Brasileira de Educação Médica, Campinas, v.41, n.1, p.134-144, 2017.

CASTILLO, A. R. G. L.; RECONDO, R.; ASBAHR, R. F.; MANFRO, G. G. Transtornos de Ansiedade. Revista Brasileira de Psiquiatria, São Paulo, v.22, s.2, p.1-4, dez. 2000.

CAVALCANTE, F. C. A. R.; PSCHISKI, P. H. Aplicabilidade da musicoterapia pontual sobre a ansiedade decorrente da hipóxia isquêmica encefálica experimental. 2015. 34 f. Trabalho de Conclusão de Curso (Graduação em Fisioterapia) - Centro Universitário de Brasília, Brasília, 2015.

CUNHA, R.; VOLPI, S. A prática da musicoterapia em diferentes áreas de atuação. Revista Científica/ FAP, Curitiba, v.3, n.1, p.85-97, jan./dez. 2008.

FRANCO, M.; RODRIGUES, A. B. A música no alívio da dor em pacientes oncológicos. Einstein, v.7, p.147-151, 2009.

FIRMEZA, M. A. et al. Uso da música no controle da ansiedade em ambulatório de cabeça e pescoço: ensaio clínico randomizado. Revista da Escola de Enfermagem da USP, São Paulo, v.51, 2017.

HADDAD, M. L.; OLIVEIRA, M. M. B. de; SIMOES, L.; MARCON, S. S. Acupuntura em mães lactantes de recém-nascidos de muito baixo peso: um relato de experiência. Ciência, Cuidado e Saúde, Maringá, v.8, n.1., p.124-130, jan./mar.2009.

KUREBAYASHI, L. F. S. et al. Auriculoterapia para redução de ansiedade e dor em profissionais de enfermagem: ensaio clínico randomizado. Revista Latino-Americana de Enfermagem, Ribeirão Preto, v.25, 2017. 
KUREBAYASHI, L. F. S.; FREITAS, G. F. de; OGUISSO, T. Enfermidades tratadas e tratáveis pela acupuntura segundo percepção de enfermeiras. Revista da Escola de Enfermagem da USP, São Paulo, v. 43, n. 4, p.930-936, dez. 2009.

JUNIOR, F. E. L. P. et al. Influência da Música na dor e na ansiedade decorrentes de Cirurgia em Pacientes com Câncer de Mama. Revista Brasileira de Cancerologia, v.58, n.2, p.155-141, dez. 2012.

LIN, C. A.; HSING, W. T.; PAI, H. J. Acupuntura: uma modalidade terapêutica validada no arsenal terapêutico do médico atual. Revista de Medicina, São Paulo, v.85, n.3, p.110-113, jul./set. 2006.

OLIVEIRA, G. C. de. et al. A contribuição da musicoterapia na saúde do idoso. Cadernos UNIFOA, Três Poços, v.2, n.20. p.85-94, dez. 2012.

ROCHA, S. P.; BENEDETTO, M. A. C. de; FERnANDEZ, F. H. B; GALLIAN, D. M. C.; A trajetória da introdução e regulamentação da acupuntura no Brasil: memórias de desafios e lutas. Ciência e Saúde Coletiva, Rio de Janeiro, v.20, n.1, p.155-164, jan. 2015.

SANTOS, F. A. S; GOUVEIA G. C.; MARTElli, P. J. L.; VASCONCELOS, E. M. R.; Acupuntura no sistema único de saúde e a inserção de profissionais não-médicos. Revista Brasileira de Fisioterapia, São Carlos, v.13, n.4, p.330-334, jul./ago. 2009.

SILVA, A. L. P. da. O tratamento da ansiedade por intermédio da acupuntura: um estudo de caso. Psicologia: Ciência e Profissão, Brasília, v.30, n.1, p.200-211, 2010.

SILVA, E. D. C. da; TESSER, C. D. Experiência de pacientes com acupuntura no Sistema Único de Saúde em diferentes ambientes de cuidado e (des)medicalização social. Cadernos de Saúde Pública, Rio de Janeiro, v.29, n.11, p.2186-2196, nov. 2013.

STALL, P. et al. Efeitos do método Rolfing de integração estrutural e da acupuntura na fibromialgia. Revista Dor, São Paulo, v.16, n.2, p.96-101, jun. 2015.

TAKIGUCHI, R. S. et al. Efeito da acupuntura na melhora da dor, sono e qualidade de vida em pacientes fibromiálgicos: estudo preliminar. Fisioterapia e Pesquisa, São Paulo, v.15, n.3, p. 280-284, set. 2008.

TUDISSAKI, S. E. Ensino de música para pessoas com deficiência visual. 2014. 167 f. Dissertação (Mestrado em Música) - Universidade Estadual Paulista, São Paulo, 2014.

UTIDA, L. R. da S. Os efeitos da musicoterapia nos níveis de ansiedade e de estresse em portadores de válvula cardíaca. 2015. 89 f. Dissertação (Mestrado em Música) - Universidade Federal de Goiás, Goiânia, 2015.

VIANnA, M. N. S; BARbOSA, A. P.; CARVAlhaES, A. S.; CUNHA, A. J. L. A. A musicoterapia pode aumentar os índices de aleitamento materno entre mães de recém-nascidos prematuros: um ensaio clínico randomizado controlado. Jornal de Pediatria, Rio de Janeiro, v.87, n.3, jan. 2011.

ZANINI, C. R. de O. et al. O Efeito da Musicoterapia na qualidade de vida e na Pressão Arterial do Paciente Hipertenso. Arquivos Brasileiros de Cardiologia, São Paulo, v.93, n.5, nov. 2009. 